\title{
The measurement of interpersonal interactions with continuous spatiotemporal data: Application to a study of the effects of resource competition on racial group interactions
}

\author{
John Tawa ${ }^{1} \cdot$ Rosalyn Negrón ${ }^{2} \cdot$ Jürgen Pfeffer $^{3}$ \\ Published online: 3 September 2019 \\ (C) The Psychonomic Society, Inc. 2019
}

\begin{abstract}
We describe a sequential qualitative $\rightarrow$ quantitative mixed-method procedure used to construct conceptually grounded quantitative metrics of interpersonal behavior from continuous spatiotemporal data. Metrics were developed from data collected during an experiment in which racially diverse participants interacted with self-resembling avatars at social events hosted in the virtual world Second Life. In the qualitative stage, the researchers conceptualized four distinct patterns of movement from overhead video recreations of participants interacting during the social events. In the quantitative stage, these patterns of movement were operationalized into metrics to reflect each type of observed interpersonal behavior. The metrics were normalized through a series of transformations, and construct validity was assessed through correlations with self-report measures of intergroup behavior. Finally, the metrics were applied to an analysis of the virtual-world study examining the influence of resource competition on racial group interactions. The findings contribute to our understanding of the influence of resource competition on Blacks', Asians', and Whites' group dynamics. Applications of these metrics for the future of the psychological study of interpersonal behavior are discussed.
\end{abstract}

Keywords Dynamic spatiotemporal data $\cdot$ Group dynamics $\cdot$ Race $\cdot$ Mixed methods

The American Psychological Association (APA) declared the first decade of the 21 st century as the "decade of behavior," a response to the recognition that research examining actual behavior - in lieu of self-report behavior-had declined dramatically since the 1970s. Within intergroup psychology, studies examining groups of people interacting in real time have declined considerably, once exemplified by studies such as Muzafer Sherif's Robber's Cave experiment and Philip

The authors would like to thank the following people: Fred Morstatter for his assistance in creating the short video re-creations of the virtual social events; Marcos Anello for development of the data collection script within Second Life; and Alison Leung for her graphic design in Figure 1. Questions and comments concerning this article should be addressed to the first author, John Tawa, at jtawa@mtholyoke.edu.

John Tawa

jtawa@mtholyoke.edu

1 Mount Holyoke College, South Hadley, MA, USA

2 University of Massachusetts, Boston, MA, USA

3 Bavarian School of Public Policy, Technical University of Munich, Munich, Germany
Zimbardo's Stanford Prison study. A review of the literature within social psychology suggests that as a discipline, we have not adequately responded to APA's call (Baumeister, Vohs, \& Funder, 2007). New technological developments might help fill this void, and in particular, might enable a breadth of research linking people's physical movement in space during real-time interactions with their psychological tendencies. Global positioning satellite (GPS) and Bluetooth sensors increasingly enable researchers to collect virtually continuous streams of data regarding how people physically relate in space over an occurrence of time (i.e., spatiotemporal data), and such data could offer researchers an opportunity to return to a focus on live behavioral interactions with greater sophistication than ever before. Understanding how to analyze spatiotemporal data with empirical rigor beyond direct observation could be instrumental in increasing the number of realtime behavior studies within intergroup psychology.

We speculate there are two reasons why psychology has hesitated to incorporate technology-based spatiotemporal data. The first is a current absence of meaning making around interpersonal spatial patterns of movement that would convince social and intergroup psychologists of the applicability of these metrics to their research questions. For example, 
movement toward and away from others over time is relatively easy to compute, yet, physical proximity can have multiple meanings; we approach people when we feel kinship, but also approach people when we feel confrontational (e.g., Ashton, Shaw, \& Worsham, 1980). Thus, one goal for the present research was to induce a variety of metrics of interpersonal spatial behavior that are more nuanced than physical proximity and that are grounded in qualitative observation of patterns of movement and interaction among multiple participants and their corresponding verbal content.

A second challenge for psychologists may be the lack of understanding about how to operationalize interpersonal behaviors by reducing oftentimes voluminous streams of spatiotemporal data into useable metrics that could, for example, be correlated with standard survey measures that are more familiar to psychologists (e.g., measures of intergroup anxiety) and/or function as an outcome variable on which group differences can be examined (e.g., gender differences in interpersonal distance over time). Simultaneously, metrics should be computed in a way that does not result in significant losses of data. Thus, a second goal for this research is to offer calculation procedures for metrics of interpersonal behavior derived from continuous spatiotemporal data while minimizing the loss of data.

We specify three dimensions that characterize spatiotemporal data - interval versus continuous-time data, macro- versus microspatial data, and sociocentric versus egocentric perspective data - and suggest that although continuous-time, microspatial, and egocentric data will likely be of the greatest use and interest to intergroup psychologists, data of this quality have rarely been collected. Below, we use this framework to review previous spatiotemporal research.

\section{Studies examining spatial behavior across time}

Social science researchers are increasingly collecting spatiotemporal data in order to understand patterns of growth and change in, as well as the influence of physical space on, humans' social relationships over time. People's movement in physical space has been assessed through various mechanisms including: geocoded GPS data (Ákos, Beck, Nagy, Vicsek, \& Kubinyi, 2014; Eagle, Pentland, \& Lazer, 2009), smartphone tracking applications (Aharony, Pan, Ip, Khayal, \& Pentland, 2011; Cho, Mayers, \& Leskovec, 2011), signal transponders (Frencken, Lemmink, Delleman, \& Visscher, 2011), overhead camera stills (Duarte et al., 2012; Passos et al., 2009), and analogously through virtual reality devices and in virtual worlds (e.g., Dotsch \& Wigboldus, 2008; Friedman, Steed, \& Slater, 2007; Tawa, 2017; Tawa, Negrón, Suyemoto, \& Carter, 2015; Toppenberg, Bos, Ruiter, Wigboldus, \& Pryor, 2015). Given the wide variety of technologies, each with unique capacities for collecting spatiotemporal data, applications of these technologies to research questions range considerably.

In the following review, we examine the body of research using spatiotemporal data. We organize our review along the three dimensions of interval versus continuous-time data, macro- versus microspatial data, and egocentric versus sociocentric perspective data. For purposes of classification within this review, continuous-time data include spatial location data assessed at intervals of 30-s or less; microspatial data includes data that is able to capture distances between interacting nodes within distances of 5-m or less; and egocentric perspective data includes data that enable analyses of the activity and potential of each individual node rather than the group as an entity. We believe that this quality of data will be of particular interest and use to social and intergroup psychologists. Snapshots of people's spatial locations within a networkwhich is characteristic of interval time data - can radically misrepresent the true relational dynamics of the community members, which can reveal themselves through more continuoustime data (Tantipathananandh, Berger-Wolf, \& Kempe, 2007). Moreover, psychologists recognize that meaningful interpersonal interactions occur within a relatively constrained (i.e., microspatial) social space (e.g., Ashton et al., 1980). Finally, social and intergroup psychology, by definition, examines the experiences of individual actors existing within a group (Allport, 1985); thus, metrics should represent the individual level (i.e., egocentric data) that could, for example, be correlated with standard survey measures of personality characteristics.

Interval versus continuous-time data A distinction can be made between interval and continuous-time data (Tantipathananandh et al., 2007). Interval time data include multiple spatial location assessments taken from participants at discrete time points in a longitudinal study. Cho et al. (2011), for example, followed participants for approximately 2 years and deduced the spatial location of the participants on the basis of the nearest cell phone tower each time the participant generated or received a call, and also from the recorded geolocations each time a participant generated a "check-in" from their mobile phone on two social network websites (i.e., Gowalla and Brightkite). Intervals may also be regularly spaced, rather than "per-incident" (e.g., cell phone calls), and the timing of intervals between spatial assessments range broadly; for example, spatial assessments have been assessed at 5-min (Eagle et al., 2009), 15-min (Silm, Ahas, \& Nuga, 2013), and 30-min (Benkert, Gudmundsson, Hübner, \& Wolle, 2008) intervals.

Some researchers using interval time data collection methods have made use of analytic approaches that computationally simulate and impute data to account for time points between assessment periods (Dijkstra, Cillessen, \& Borch, 2013; Mercken, Steglich, Sinclair, Holliday, \& Moore, 2012; Sijtsema et al., 2010; Steglich, Snijders, \& West, 2006; Woolf, Potts, Patel, \& McManus, 2012). Most recently, and most promisingly, Long 
and Nelson (2013b) have developed a kinematic interpolation method that takes into account the velocity and acceleration of a node to more accurately deduce spatial locations between interval time assessments. Despite the improvement of imputed data to account for missing data, processes that occur within these time lapses are nonetheless inferred and not directly assessed.

We consider assessments of spatial locations to be continuous when they are computed in intervals of $30-\mathrm{s}$ or less. Saelens, Moudon, Kang, Hurvitz, and Zhou (2014), for example, used GPS devices and accelerometers - both triggered at 30-s time intervals - to infer a log of participants' bouts of physical activity over the course of seven days. Even more acutely, Duarte et al. (2012) analyzed the positioning of soccer players (e.g., centroids of each team) during scoring plays, by computing positional data at freeze frames of video footage of the soccer match at 0.04-s intervals.

Although interval time data may be adequate for long-term behavioral processes, such as the migratory patterns of birds over the course of a season, intergroup psychologists are likely to be more interested in interactions occurring between people in a relatively constrained period of time, for example, people's social behavior at a social gathering that lasts a few hours. Consider, for example, a person who is quite social but who occasionally steps away from the crowd to look at some artwork or other décor in the environment. An interval time analysis of this person's spatial behavior - say four recordings of spatial locations at 30-min intervals - could, by chance, capture this person alone and away from the group on one or more occasions, and thus could result in an underestimation of this person's sociality. A more continuous-time analysis of 30-s intervals would be much more likely to record this person's active social behavior more accurately.

Macro- versus microspatial data Although a relatively sizable body of work within social network analysis (SNA) and geographic information systems (GIS) has examined "macro" spatial patterns, such as tracking the movements of people throughout the city during a workday, significantly less research has examined "micro" spatial patterns, such as people's tendencies to approach and avoid each other within a shared social setting. The latter, of course, requires considerably more advanced technology with the capacity to capture interactions occurring as close as one foot/meter, and will be the method of choice for psychologists who are interested in interpersonal interactions. Macrospatial data include spatiotemporal data collected on a larger spatial scale, and for the purpose of this article, are defined as occurring within distances between tracked actors at 5-m or greater. Such data are suitable for research questions concerning people's movement and interaction patterns over a large surface area such as a park or city (e.g., Zhang \& Chai, 2011). For collection of macrospatial data, a number of sufficient technologies are readily available such as GPS geocoding and Bluetooth scanners. A number of these studies have gleaned location-based data from users' own cell phones (e.g., Cho et al., 2011; Eagle et al., 2009) or by distributing similar devices to participants (e.g Saelens et al., 2014). Although the spatial accuracy of these devices is limited, they are easily accessible and cost-effective, and are sufficient for research questions concerning macrospatial movement patterns. Cho et al. (2011), for example, used location-based data provided by cell service providers that located initiated and received phone calls with a spatial accuracy of approximately $3-\mathrm{km}$. Although this range is large, such devices would be sufficient for research questions related to people's movement in a large social space, such as a city. As a more precise option, Eagle et al. (2009) provided participants with smartphones programmed with a Bluetooth-based scanning application that detected the presence of other participants (running the same application) within a 5- to 10-m range. Although the Bluetooth software used in Eagle and colleagues' study offers greater acuity than previous studies described above, interpersonal distance thresholds of 5- to 10-m range would still not be sufficient for analysis of interaction patterns that would likely be of interest to social and intergroup psychologists. Classic research within social psychology, for instance, has established that casual and public interactions typically occur within a range from 30 in. to 30 feet (approximately $9-\mathrm{m}$ ). Within this range, closer distance to others tends to communicate affinity and warmth toward others, whereas farther distance communicates distrust or dislike (Ashton et al., 1980; Evans \& Howard, 1973; Hall, 1964; Mehrabian, 1969). Moreover, cultural norms in interpersonal distancing may be just centimeters or inches in difference, but they are nonetheless significant (Sorokowska et al., 2017). Thus, location-based technologies will likely only be of interest to intergroup psychologists if they are able to determine distances within a minimal range of 5-m, again, what we refer to as microspatial data.

Considerably less research exists that can assess microspatial patterns, or interactions between people occurring within a range of 1-5-m (Ákos et al., 2014; Duarte et al., 2012; Frencken et al., 2011), undoubtedly a reflection of the greater technological demand required for such analyses. Some available tools are capable of assessing microspatial patterns. Inmotio Object Tracking uses Bluetooth technology and was developed for tracking the movements of members of sports teams in play. Base stations placed on the athletic field transmit signals sent to and from athletes wearing transponders. The transponders have a location accuracy of 20-30-mm (Frencken et al., 2011). Ákos et al. (2014) used "custom designed high resolution GPS" devices with a spatial accuracy of $1-2-\mathrm{m}$ to track the patterns of movement among alphas dogs and their pack. Although these technologies are available, we are not aware of any research specific to studying interpersonal behaviors that has adopted them. Currently this body of literature appears limited to examining animal movement/behavior (e.g., Ákos et al., 2014) and sporting behavior (e.g., Duarte et al., 2012; Frencken et al., 2011; Passos et al., 2009). 
Egocentric versus sociocentric perspective data Finally, we make a distinction between egocentric and sociocentric perspective data, and suggest that the majority of spatiotemporal research has collected and analyzed behavior sociocentrically. Sociocentric perspectives examine the group as a whole, describing, for example, the coordinated movement of a group (Duarte et al., 2012; Frencken et al., 2011), the surface area covered by members of a group as a collective (Duarte et al., 2012), the emergence of a subgroup or flock (Benkert et al., 2008), and the development of a single leader within a group (Ákos et al., 2014; Andersson, Gudmundsson, Laube, \& Wolle, 2008). For example, in Duarte et al.'s (2012) study of scoring plays during a soccer match, the surface area covered by the attacking team was found to be significantly larger at the moment of the assisted pass than the surface area of the defending team. Andersson et al. (2008) developed an algorithm for detecting an emerging leader from a group of nodes based on a minimum time threshold in which one entity is positioned ahead of other entities. In both examples, the unit of analysis was the group; metrics pertain to the area of coverage of the group and the emergence of a group leader.

Egocentric perspectives, by contrast, examine the potential or tendencies of each individual actor behaving within the context of a group. We emphasize the latter clause because a number of studies have examined individual spatiotemporal behaviors but do not necessarily do so in relation to a group (Hurvitz, Moudon, Kang, Saelens, \& Duncan, 2014; McArdle, Demsar, van der Spek, \& McLoone, 2014). McArdle et al. (2014), for example, used GPS data and 3-D visualizations to determine a variety of individual movement patterns exhibited by pedestrians, including route preferences, window-shopping (characterized by slow, steady forward movement), and café stops (characterized by long pauses in movement). Although these are examples of individual behavior assessed through spatiotemporal measures, they are not necessarily behaviors that occur within the context of group interactions.

Egocentric data are collected from the perspective of each actor within a group (e.g., what is each individuals' degree, tendency, or potential to emerge as a leader?), rather than from the perspective of the group as a whole (e.g., which individual will emerge as the leader of this group?; Long \& Nelson, 2013a). As one example of egocentric data, Passos et al. (2009) analyzed the dyadic movements of rugby players during scoring plays in which a single attacker approached a try line while a single defender attempted to obstruct. Using an approach similar to that of Duarte et al. (2012), Passos et al. (2009) used 0.04-s stills gleaned from video footage of the dyadic interactions and observed that decreases in interpersonal distances between the attacker and defender resulted in changes in the angle at which the attacker approached the goal line. Moreover, these sudden changes in angles caused greater instability (more variation) in the dyadic system (collective movement between attacker and defender), and greater variation in the system was more likely to result in an attempted tackle. Thus, the unit of analysis was from the perspective of the attacker (i.e., the angle at which they approached the try line); however, the defender's movement dynamically influenced the attacker's movement. For intergroup psychologists, the goal would be to develop metrics of individual behavior existing in a context of interactions with others.

In this article we report on a sequential qualitative $\rightarrow$ quantitative mixed-method procedure (Creswell \& Clark, 2017) for developing metrics of interpersonal behavior from spatiotemporal data that are grounded in observed interactions. Our metrics are derived from data collected within the virtual world Second Life, during an experiment in which Black, Asian, and White participants created self-resembling avatars and interacted in social events modeled to simulate contexts of resource competition. The virtual-world environmentreplete with user data - provided an ideal medium for exploring and assessing continuous, microspatial, egocentric data. Understandably, there are questions about the validity of virtual-world behavior as analogues of real-life behavior (Williams, 2010). We address these questions in the next section, before describing our procedure for metric development.

\section{Behavioral research using virtual technology}

Various studies of human behaviors have used virtual technology, including desktop-accessed virtual environments (DVEs) such as Second Life, and immersive virtual environments (IVEs) such as virtual-reality headsets that are worn by a participant (Friedman et al., 2007). In DVEs participants operate an avatar on a computer screen - similar to a game character in a video game - typically from a first-person perspective or from a third-person perspective with the "point of view" directly behind the avatar. In IVEs, a participant's real-life movements are tracked and represented in the virtual environment, including the movement of limbs; however, full-body directional movement is often limited to a short walk in any one direction, as they occur within a confined area (e.g., a small room or cubicle; Toppenberg et al., 2015). In this section we address the question of the validity of virtual environments for the study of real-life behavior and then describe in more detail the findings of our previous study in Second Life (i.e., Tawa et al., 2015), which was limited by a reliance on physical distance as a behavior measure, and which thus served as an impetus for the development of metrics of more specific patterns of movement.

The validity of virtual technology for the study of human behavior Studies using both DVEs (Eastwick \& Gardner, 2009; Feldon \& Kafai, 2008; Friedman et al., 2007; Hasler \& Friedman, 2012; Lee \& Park, 2011; Lo, 2008; Tawa, 2017; 
Tawa et al., 2015; Vang \& Fox, 2014; Yee \& Bailenson, 2008; Yee, Bailenson, Urbanek, Chang, \& Merget, 2007) and IVEs (Bailenson et al., 2004; Bailenson, Blascovich, \& Guadagno, 2008; Blascovich et al., 2002; Dotsch \& Wigboldus, 2008; McCall, Blascovich, Young, \& Persky, 2009; Toppenberg et al., 2015) tend to find that people's virtual behavior is similar to people's behavior in real life. As examples, research using the DVE Second Life has found that, as in the real world, avatars were inclined to have less eye contact with unpleasant users (Yee et al., 2007), and that avatars tend to hold realistic interpersonal distances during interactions, initiating interactions at approximately 4-5 virtual meters, and moving closer $(1-1.5-\mathrm{m})$ for more personal engagement (Friedman et al., 2007). Using IVEs, research has shown that, similar to real life, people tend keep greater distance from people with stigmatized identities but also from people whom they perceive as having higher status. In one study, participants approached a hospital bed more slowly if a patient was diagnosed with HIV or cancer than for those with a broken leg, and also kept larger distance from HIV patients than from cancer patients (Toppenberg et al., 2015). In another study, participants tended to approach a "stranger" avatar more closely than a "tutor" and, as in real life, retained greater personal space toward people with higher social status (Bailenson et al., 2004).

Also consistent with real life, and relevant to our research, people tend to display prejudicial behavior toward minority avatars in virtual environments (Dotsch \& Wigboldus, 2008; Eastwick \& Gardner, 2009; Lee \& Park, 2011; McCall et al., 2009; Tawa, 2017; Tawa et al., 2015; Vang \& Fox, 2014). For example, in a study using a DVE, online avatars were more likely to comply with a request for a favor when approached by a researcher piloting a White avatar than by one piloting a Black avatar (Eastwick \& Gardner, 2009). In a study using an IVE, native Dutch participants experienced greater physiological stress when approaching a Moroccan (i.e., minority) avatar than when approaching a White avatar (Dotsch \& Wigboldus, 2008). Among these studies using virtual technology, spatiotemporal movement has been limited to relatively rudimentary metrics, such as physical distance and speed of approach (Bailenson et al., 2004; Dotsch \& Wigboldus, 2008; Friedman et al., 2007; McCall et al., 2009; Tawa et al., 2015; Toppenberg et al., 2015). We faced these limitations in a previous analysis of our Second Life data (i.e., Tawa et al., 2015); here we return to these data in an effort to develop more specific metrics of interpersonal behaviors. For the present analysis DVEs are an ideal technology, because of the more limitless physical movement available.

The effects of resource competition on intergroup distance in Second Life In this dataset we used the DVE Second Life to collect coordinate data at 1-s time intervals as participants interacted with other participants' self-resembling avatars in 15-min social events. The social events were hosted in a parcel on a private island in Second Life that featured an outdoor patio overlooking a lake and an attached gazebo. The purpose of our study was to examine how resource competition - the real or perceived sense that the allocation of resources to one person or group inevitably means less resources for other people or groups - affected racial group members' interactions. Some participants were randomly assigned to interact in events designed to simulate resource competition $(N=55){ }^{1}$ whereas others interacted in "utopian" social events in which no resource competition was present $(N=68)$. In the resource competition setting, participants were asked to imagine that they were local business leaders and were tasked with assembling a team of five of their peers; participants were led to believe that the person who formed a team of the "hardest working" and "most intelligent" people could maximize their chance of winning a \$300 gift certificate to Amazon.com. The participants in the utopian condition were also tasked with assembling a group of five peers, but they were not led to believe that the merits of their group members increased their chances of winning the gift certificate. In actuality, in both conditions one participant was randomly selected for the $\$ 300$ gift card award. To create groups, participants sent "friend requests" to other participants, and shared group membership was only confirmed once the friend request was accepted by the recipient. We were interested solely in the processes of formation of the groups; participants did not complete any further tasks once the groups had been formed.

In a previous study of these data (Tawa et al., 2015), we hypothesized that racial group members would have greater increases in physical distance over time while creating groups in the resource competition settings relative to the utopian settings. What we found was that changes in physical distances over time were not linear; within resource competition settings, racial groups' physical distance toward each other actually initially decreased (during the first $5 \mathrm{~min}$ of the event), but then began to increase over the duration of the event (during the last $10 \mathrm{~min}$; Tawa et al., 2015). Despite the partial support of our hypotheses, we faced some limits in our ability to interpret the findings, due to the reliance on physical distance as a metric of interpersonal interactions. For example, it wasn't clear to us why racial groups initially approached each other in the resource competition settings. The impetus for the development of the present metrics was at least in part to help us understand in a more nuanced manner how resource competition affected interpersonal interactions and intergroup relations. In what follows, we turn to a description of our

\footnotetext{
${ }^{1}$ In the original study, there were two different types of resource competition-merit-based and an insider-based resource competition; however, given questions about the operationalization of insider-based resource competition and a lack of significant findings, the participants assigned to the insider-based condition were dropped from the present analysis. See Tawa et al. (2015) for more detail.
} 
approach for developing metrics of interpersonal behavior that are conceptually grounded in qualitative analyses of the participants interacting during these events.

As has been recommended by recent reporting standards for mixed-method research (Levitt et al., 2018), our methods and results are organized to reflect the chronological order in which the analyses were conducted. First, we describe the qualitative procedures used to generate four distinct patterns of participants' interactive movement (method) and give an in-depth description of the patterns of movement (results); next we describe the quantitative operationalization of these patterns of movement into metrics, an assessment of the normality and validity of the metrics, and finally the application of the new metrics to a study of the effects of resource competition on intergroup relations.

\section{Qualitative methods and results}

Our qualitative data analysis included a data visualization strategy that provided us with an overview of participants' patterns of movement and corresponding chats, as well as analyses drawing from ethnographic methods of these videos. In our results, we describe our observations and related conceptualizations of four distinct participant movement patterns.

\section{Data analysis}

Data visualization One common approach for analysis of continuous interpersonal space-time data involves the production of complex data visualization maps that enhance analysts' ability to find patterns in movement data (Andrienko \& Andrienko, 2007). The visualizations can be a precursor or complement to the quantification of movement, including metric development. GIS scientists have been particularly productive in modeling, classifying, and quantifying movement patterns applicable to the study of individual behavior as well as group dynamics (Dodge, Weibel, Ahearn, Buchin, \& Miller, 2016; Dodge, Weibel, \& Lautenschütz, 2008; Long $\&$ Nelson, 2013a). Working from enormous databases of timestamped and geo-coded data points, movement pattern researchers have developed ways to detect a range of behaviors, including leader following (Andersson et al., 2008; Laube, Imfeld, \& Weibel, 2005), flocking/crowd dynamics (Benkert et al., 2008), and avoidance (Noyon, Claramunt, \& Devogele, 2007).

In the present study, we used participants' coordinate data to re-create JavaScript videos depicting an overhead view of participants' patterns of movements on a two-dimensional space. The chat logs, which were timestamped, were matched to the timestamped coordinate data so that the chats displayed with a timing that corresponded with the movements playing out in the movies. In contrast to movement pattern research working with GPS data or with computer simulations, our present study had the key benefit of tracking movement across time in a visually small and relatively contained virtual space. This made it possible to see "birds-eye" overviews of individual and interactive behavior. The "birds-eye" videos facilitated the qualitative analysis of both the movement of the group as a whole and the movement of each individual participant. Text $\operatorname{logs}$ of chats between participants enhanced our ability to understand the antecedents and consequences of people's movement in relation to others.

A total of six social events (three resource competition and three utopian condition events) from the Second Life study (Tawa et al., 2015) were visualized in the movie recreations. Nodes were used to represent the participants in the twodimensional space, each with a unique ID number. Node colors corresponded to participants' race-Asian, Black, and White - and there were no additional identifiers, such as those related to gender. A real-time version of the movie showed spatiotemporal movements for every second of the trial. A second set of movies displayed movements approximately 10 times faster ("fast"), and a third set of movies displayed movements approximately 100 times faster ("very fast"). A fourth set of movies traced the lines for the paths taken by all participants in each of the trials.

Analytical approach Each movie was first viewed at fast and very fast speeds, to gain an overview sense of the dynamics of the individual and group interactions, from beginning to middle to end. Consistent with qualitative methods such as the grounded-theory method, our analysis proceeded iteratively with no predetermined hypotheses (Glaser, Strauss, \& Strutzel, 1968). Instead, we allowed observable patterns of movement to emerge inductively (Creswell \& Clark, 2017; Glaser et al., 1968). We took first-impression notes about the overall group processes, notable patterns, and any evidence of racial preference in spatiotemporal arrangements. Next, the complete chat log was read, to better understand the relationship between individual and group movements and the conversational agency of the participants. The chat logs, for example, revealed participants' impressions of the experiment, their greetings and "chit-chat," their strategies for completing the task set forth by each condition, any confusion and questions about experiment process, and key conversational topics. Observations from the chat logs were noted along with notes from the movies.

After we had read the chat log, each movie was viewed again while matching the chat activity with the movement of individual nodes. Participants who were particularly vocal or who seemed to encourage group formation in their conversations were located in the movie, to gauge the extent to which the spatiotemporal arrangements were influenced by their words and actions. When clusters of nodes formed, IDs of the members of each cluster were matched to chat log activity, 
to understand antecedents for the cluster formation and to map the relationship between the conversations and any changes over time in the clusters.

We cross-checked key findings from our initial observations with the real-time movies, which afforded more time for careful observations of movements and interactions. The movies that traced the lines of the paths taken by each of the participants were useful for getting a handle on the level of spatiotemporal movements in each trial and for identifying areas of significant overlapping activity. Such visual markers furthered our ability to identify patterns that we could then describe through algorithms. This iterative, multipronged analytical approach yielded insights about key patterns that could be recognized across the trials. Our intent for this level of nuance in observation was to maximize the likelihood that our metrics would be grounded in actual social interactional dynamics observed in virtual space.

\section{Qualitative results}

In this article, we propose the use of qualitative assessments as precursors to the development of formal definitions and algorithmic procedures to model the evolution of real-time groups. A number of metrics emerged from our qualitative assessments of real-time interactions in a virtual space, some of which are related to existing measures, and others point to promising new research avenues. Thus, we understand our metrics as complements to existing clustering algorithms and other measures of subgroup formation and maintenance. We induced a total of four distinct patterns of movement from our analyses. These included leadership, seeker, marginality, and diversity/homophily. In the following sections, we describe each observed type of behavior and describe any previous research that has attempted to quantify similar types of behaviors.

Leadership Previous work that aimed to understand the influence of leader-following on simulated crowd behavior began by first finding potential leaders using standard centrality measures and setting "following probabilities" that an individual would follow a leader who appeared within their visualspatial field (Li \& Lin, 2011). Others have applied datamining algorithms to GIS databases in order to match motion patterns that indicate "trend setting" (Dodge et al., 2008; Laube et al., 2005). Quite simply, in trend setting, an actor reproduces the trajectory of another actor who moved seconds, minutes, or days before. Once detected, such leading behavior requires further interpretation. A leader or trendsetter may be initiating group behavior or may just be spatially configured in front of other actors (Andersson et al., 2008). Our own approach was to attend to the various ways participants demonstrated the ability to have others follow them within Second Life's space. We also paid close attention to chat log evidence that a given person was indeed influencing others' behaviors by what they said. The excerpt below is an example of the sort of commentary that, in conjunction with observed movements, pointed to leadership behavior, particularly by "LcKy13.",

[16:14] LcKy13: all of you look pretty smart to me. I think if we all join up into one big team we can accomplish huge things and bring this town out of its recession [16:15] alvamp92: can i join ur group

[16:15] Annie001: kmm819, let's work together!

[16:15] DaSilveira (loira2569):

ok well $i$ am stuck somehow

[16:16] DaSilveira (loira2569): obviously video games

are not my strong suit

[16:16] Annie001: Mine either!

[16:16] alvamp92: lcky13 can i join ur group

[16:16] LcKy13: anybody can join my group

[16:16] Botman4004: Ok im me to learn what $i$ can

bring to the table

[16:16] jtaznboy123: hey do you guys mind if $i$ work with you guys

[16:16] alvamp92: well im joinging u group

[16:17] LcKy13: everybody who is in meet me in the

back left corner and we will discuss our business in further detail

Given our focus on egocentric variables, which focuses on an actor but recognizes that the actor exists within a context of many other interacting agents, grasping follow-the-leader dynamics required attention to the behaviors of followers, who often play a significant role in escalating phase transitions (cf. Rogers, 2003, for a discussion of follower dynamics), such as the development of the group led by LcKy13 depicted in the excerpt above. That being said, follow-the-leader dynamics were not visibly apparent in all trials. Moreover, the level of influence varied among the observed leaders; for example, some influenced the movement of just one other person, and others initiated the development of a subgroup. In our leadership metric, we aimed not simply to categorically identify "leaders," but to capture the varied strengths of leadership behavior in a continuous metric.

Seeker There are few examples of previous metrics designed to capture seeking, a behavioral pattern characterized by purposeful movement between sites with a relatively brief

\footnotetext{
${ }^{2}$ In these excerpts, all timestamps, usernames, and texts are retained in their original forms, including the use of capitalization and spelling and grammar errors. The majority of our participants used their account names as their avatar names. Occasionally, a participant selected a different "display name," which is included in parentheses in the chat log. Depending on each participant's account settings, one or both names would be visible to other users.
} 
duration of time spent assessing each site. The most relevant models for this movement pattern can be found in animal ecology studies, specifically the study of foraging behavior (Bian, 2000). As with foraging for food in the wild, here seeking involved the search for a desired resource that might lead a person to move widely within a given space. In our evaluations of the movies, we flagged individual participants who moved throughout the virtual space while periodically making stops to engage others in conversation. These stops were sometimes for brief periods of time. Thus, seekers were observed moving between individuals or subgroups, apparently in search of potential contacts to achieve the trial task, as exemplified by the following exchange:

[16:11] Kimtaro: Wow I don't even know where to start

[16:11] Hippocrite: well Im gonna try to get more peo-

ple haha. Talk to you soon

[16:11] MegFischer: Same. Good luck

[16:11] Hippocrite: you too

Spatially, seekers moved frequently and covered larger surface areas of the trial grounds than did more stationary nodes. However, the seeker's movement was not independent of other nodes, and their stops in movement would almost always occur in close proximity of another node, presumably a person with whom they were interacting. Figure 1 comprises 16 screen shots taken from one of the video recreations of one of the resource competition social events. Each screen shot occurs at the first second at each of the 15-min of the trial. Node number 48 , named ImplicitFunction is indicated by a red arrow and was flagged as a seeker; this node is seen covering a wide surface area of the trial grounds and is almost always seen in close proximity of other nodes. In our operationalization of seeking behavior, we sought to capture a person's tendency to adopt a more survey-style interaction with other nodes and subgroups, talking to more people but with less depth.

Marginality Previous spatiotemporal research has attempted to model marginal or avoidant behavior (Noyon et al., 2007; Passos et al., 2009; Toppenberg et al., 2015), determined by greater physical distance from a target node (Toppenberg et al., 2015), or by a change in the trajectory of an actor in response to the approach of another node (Noyon et al., 2007; Passos et al., 2009). In our examination of the movies, we perceived some nodes that tended to skirt along the perimeter of the group or subgroups. In some of these instances, we deemed that these nodes were simply not participating in the experiment, perhaps related to technical difficulties or a loss of interest in participation. We did not consider these participants to be good representatives of marginal behavior. Instead, our interest was on participants who were actively participating in the project but tended to keep others at "arms length" or who kept interactions with others generally superficial, brief, or in a disinterested manner. In the following excerpt from the beginning of the trial, "AutumnMetaphor" does participate in the group exchange but does not initiate conversation, and tends to be relatively "matter of fact" in her responses.

\section{[16:06] ImplicitFunction: how $r$ all of you? \\ [16:06] breakers18: hi everyone \\ [16:06] ReginaPhalanges: how did you get clothes? \\ [16:06] AutumnMetaphor: Good, how are you? \\ [16:06] keitichandesu: I am well, how are you? \\ [16:06] AutumnMetaphor: Freebies \\ [16:06] gtheoo: hello \\ [16:06] AutumnMetaphor: How about you? \\ [16:06] sbdanni48: good how are you all doingg \\ [16:07] ReginaPhalanges: $i$ tried to buy them and put \\ the on. but all i meanaged to do was take them all off. . . \\ lol \\ [16:07] AutumnMetaphor: Aww \\ [16:07] ReginaPhalanges: managed* \\ [16:07] Cross2012: good, and i couldn't find any free- \\ bies really. Got stuck with these. \\ [16:07] ImplicitFunction: pretty good}

Although AutumnMetaphor's chat behavior alone would not be enough to identify her as marginalized, she does appear to be a strong example of a high level of marginality when seen in conjunction with her physical movement behavior. AutumnMetaphor (node number 44, indicated by a blue arrow) is seen in Fig. 1 as remaining on the perimeter of the group for the duration of the event. Our marginality metrics reflects the relative lack of interaction with other nodes.

Diversity/homophily For our last observation, we took information about the attributes of trial participants (i.e., race, depicted in the videos as different color nodes) in order to assess the extent to which people tended to interact with people dissimilar from themselves (diversity) or with people similar to themselves (homphily). A body of social network analysis research with longitudinal datasets supports the principle of homophily: Over time, peers tend to be attracted to and select other peers with similar visible characteristics such as race (de Klepper, Sleebos, van de Bunt, \& Agneessens, 2010; Kossinets \& Watts, 2009; van de Bunt, van Duijn, \& Snijders, 1999; van Duijn, Zeggelink, Huisman, Stokman, \& Wasseur, 2003). However, these studies - to our knowledge - have exclusively examined peer interactions through self-report assessments, rather than through direct behavioral assessments. Research in virtual environments has made some effort to assess diversity/homophily-related behaviors from continuous spatiotemporal datasets (Dotsch \& Wigboldus, 2008; Ducheneaut, Yee, Nickell, \& Moore, 2007). In the virtual- 


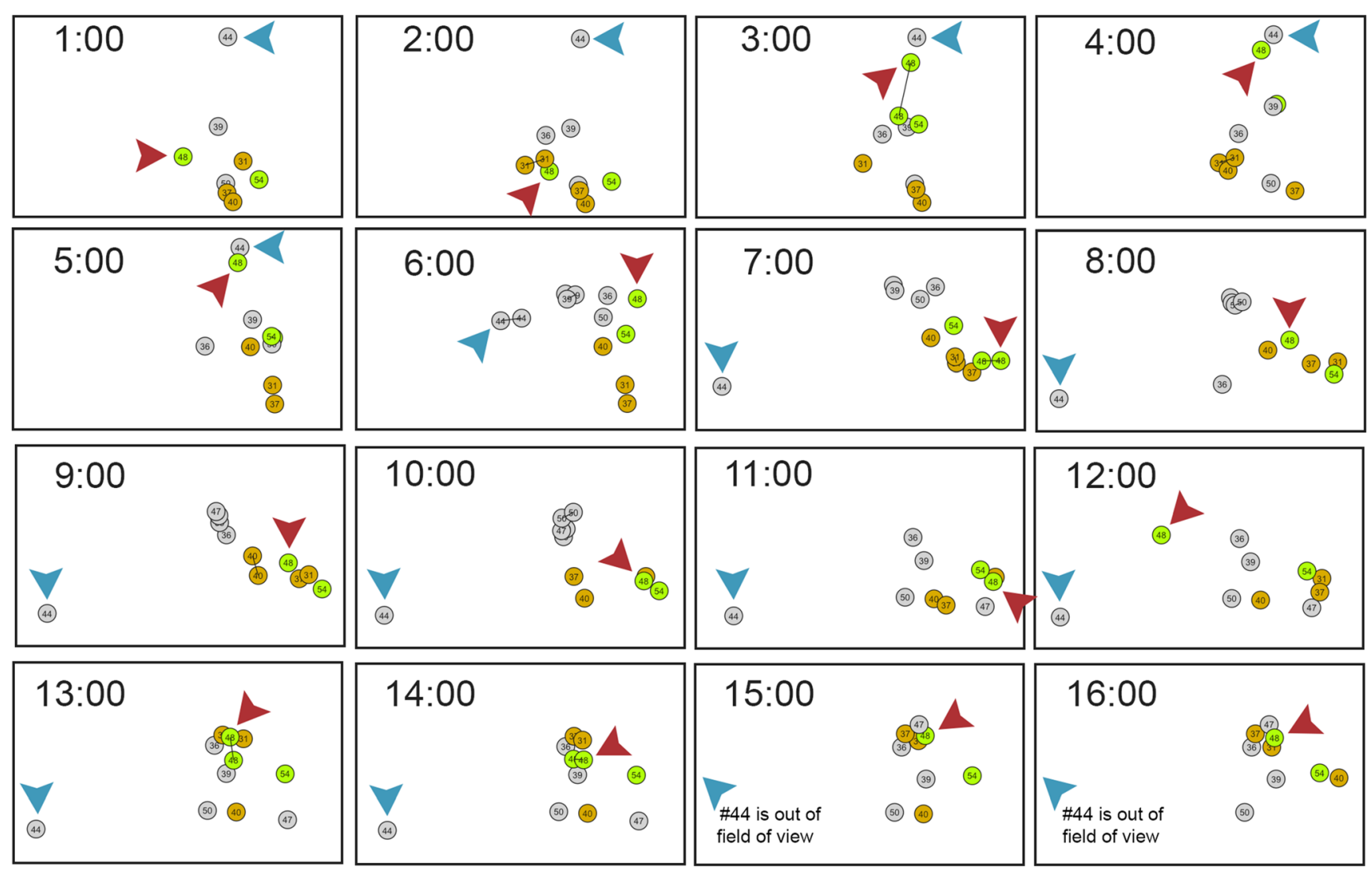

Fig. 1 Interaction among nodes in a utopian-condition trial. The red arrow indicates a seeker, named ImplicitFunction, and the blue arrow indicates a more marginal node, AutumnMetaphor

environment study described above, native Dutch (i.e., White) participants more closely approached White than Moroccan avatars (Dotsch \& Wigboldus, 2008). In another study, guilds in the massive multiplayer online game World of Warcraft were found to be more likely to survive if they comprised more diverse classes of characters, rather than more homogeneous ones (Ducheneaut et al., 2007).

Our observations of chat behaviors suggested that, particularly within the resource competition conditions - conditions in which participants believed their group member selections could lead to monetary gain - participants tended to engage other avatars with questions about their language and culture, but not directly about race. Participants may have found it more socially acceptable to ask other participants directly about their language capability rather than asking about race; nonetheless, they may have used language and culture cues to make racialized decisions about whom they deemed "intelligent" and "hardworking." For example, in the conversation below, language capacity seems to be conflated with being perceived as "smart and hardworking." Asian-languagespeaking participants such as "yi sun (stevesun)"-referred to as "steve" - appear to be particularly sought-after group members by DaSilveira and LcKy13, perhaps because of the stereotype of Asians as being inherently intelligent (Cheryan \& Bodenhausen, 2000).
[16:07] DaSilveira (loira2569): hi steve what are your credentials

[16:08] rahnny Correia (rahnny3):

are you a good worker? Tweji?

[16:08] alvamp92: hello someone

[16:08] kmm819: hi

[16:08] yi sun (stevesun): credentials?

[16:08] DaSilveira (loira2569): is anyone here majoring in business? speak more than one language?

[16:08] Botman4004: come to this corner im looking for 5 smsart individuals

[16:08] alvamp92: someone plz come to me

[16:08] meltexmex: I speak more than one language.

[16:08] emiumass08: yes i do

[16:08] yi sun (stevesun): I do

[16:08] LcKy13: yi sun i need some help with my business join my team and we can be co-owners

[16:09] emiumass08: spanish portuguese

[16:09] DaSilveira (loira2569):

great $i$ would love to work with bi-lingual and cultural people

[16:09] yi sun (stevesun): what kind of

[16:09] DaSilveira (loira2569):

$i$ speak english spanish and portuguese

[16:09] Botman4004: I can speak creole 
[16:09] meltexmex: DaSilveira, we should work together. Tell me your best qualities.

[16:09] yi sun (stevesun): I speak chinese english

[16:09] alvamp92: $i$ can speak spanish

[16:09] LcKy13: $i$ speak cold hard american english

and im bringing jobs back to the USA

[16:09] DaSilveira (loira2569): great i feel that these

people can already deal with diversity and work well

together

Similarly, later in the trial, DaSilveira deems EmiRose to be "smart" and "organized" after she discloses that she speaks basic Japanese.

[16:11] EmiRose: I can speak basic japanese

[16:11] LcKy13: i speak english

[16:11] LcKy13: most of the usa speaks english

[16:11] Annie001: Hi, I'm a very hard worker

[16:12] nora29: I can sign- like American

Sign Language.

[16:12] LcKy13: i'm a good worker a good organizer,

$i$ know how to use databases and $i$ can drive

[16:12] DaSilveira (loira2569):

emi, achou alguem mais de boa?

[16:12] Annie001: I'm also a good leader,

what are your good qualities?

[16:13] LcKy13: Who needs a job?

[16:13] alvamp92: i need a job

[16:14] yi sun (stevesun): on campus or outside?

[16:14] DaSilveira (loira2569):

emi rose you look like a smart and organized girl

[16:14] LcKy13: Let's work together.

I work with fellow co-workers

[16:14] alvamp92: campus

[16:14] DaSilveira (loira2569):

the japanese is very fundamental and useful

[16:14] LcKy13: all of you look pretty smart to me.

I think if we all join up into one big team we can accom-

plish huge things andbring this town out of its recession

In our observation of movement patterns, we could not always visually perceive race-based patterns of movement among the nodes. Yet it was possible that some degree of race-based movement patterns were occurring that were not visible to the naked eye. In some utopian trials, we did observe nodes initially coming together in race-specific subgroups (homophily), but over time they eventually began to interact with more diverse peers. For example, this pattern of race-based group movement is apparent in Fig. 1, as nodes begin to interact in more diverse groups beginning at approximately the 7 th minute of the trial. Chat logs from the resource competition trials suggest that race was in fact salient to participants' interactions. Thus, we developed a quantitative metric of diversity/homophily, reflecting the extent to which participants interacted with people racially different from themselves.

\section{Quantitative method and results}

The quantitative portion of our metric development began with operationalizing multiple variations of the four patterns of movement conceptually defined in the qualitative portion, and normality and validity analyses helped us determine which version of the metrics was most robust. In our results, we describe some preliminary findings including intercorrelations between metrics and group differences in metrics. Finally, we apply these metrics to a research problem identified in Tawa et al. (2015), in order to demonstrate the utility of these metrics for future research.

\section{Measures}

Metrics were operationalized and calculated in R. All metrics were derived from the physical location data (i.e., coordinates on the Second Life grid), which were recorded at 1-s intervals across the 15-min social events. The data represent geographic $x / y$ coordinates in a two-dimensional space. To incorporate missing or double coordinates for a specific timestamp, we aggregated the data in 5-s intervals (resulting in 180 time intervals $t$ per social event) and calculated the average position for every node for every time interval. We then calculated a Pythagorean distance matrix for all distances between each pairs of actors, based on their $x / y$ coordinates. For every time interval $t$, the distance $d$ between nodes $i$ and $j$ was defined as

$d_{i, j}^{t}=\sqrt{\left(x_{i}-x_{j}\right)^{2}+\left(y_{i}-y_{j}\right)^{2}}$.

Movement for every node $i$ was calculated between every two consecutive time intervals $t$ and $t-1$ :

$\Delta_{i}^{t, t-1}=\sqrt{\left(x_{i}^{t}-x_{i}^{t-1}\right)^{2}+\left(y_{i}^{t}-y_{i}^{t-1}\right)^{2}}$. Also, the relative distance change between every pair of actors was calculated for each consecutive time interval. All metrics were derivations of these matrices.

Leadership Our initial approach to operationalizing leadership was to simply take a cumulative score of changes in distance between each actor, in order to base the measurement on change in distance rather than on absolute or actual distance. Although this approach does not differentiate whether distances, for example, lessen because one actor moves toward others or others move toward that actor, we thought that if there were an overall actual attractive quality of a single actor, over time, others would more frequently move toward that actor, resulting in decreasing distances overall and indicating 
higher leadership behavior. Once we had executed this initial measure, we cross-checked the results with the videos to see whether those who emerged as leaders on the basis of the metric did indeed play a leadership role in the trial. We quickly realized that some actors were receiving high scores because of their leaving and then returning to groups after moving some significant distance. Instead, we observed that in several instances those who exhibited more leader-like behavior (because they indeed exerted a pull on others and/ or because of the directive quality of their chat discourse) moved rather little after some initial movements. Once they were planted, others moved toward them. We also realized that movements toward leaders were only meaningful within a relatively immediate social space; interactions occurring, for example, among members comprising an entirely different subgroup within the social event should not count toward a specific node's leader score. Thus, we revised our operationalization of leadership by rewarding actors with "points" for movement toward them by other nodes within a 5-m radius, and only while the actor remained relatively still (with a leeway of $0.5-\mathrm{m}$ movement in any direction). While an actor was moving, they did not get points for others' movement toward them. We defined the leader score $l$ for an actor $i$ as the sum of all movements toward this actor within a 5-m radius while the actor itself did not change its positions by more than $0.5-\mathrm{m}$ :

$l_{i}=\sum_{j \neq i} \sum_{t=2}^{180}\left|d_{i, j}^{t}-d_{i, j}^{t-1}\right|$ if $d_{i, j}^{t} \leq 5 \wedge \Delta_{i}^{t, t-1} \leq 0.5$.

Seeker The seeker metric should capture a person's tendency to have more frequent interactions with others, with less depth. We calculated the seeker metric by creating a matrix of the minimum distance achieved between each actor and every other node, and then computing an average of these minimum distances. We observed a potential problem with this metric operationalized in this way. In some rare instances, one participant might choose to move very little (thus not exhibiting seeker behavior), yet there might be some reason why people eventually approached them over the duration of the event. For example, others might tend to approach a node at some point in the trial in order to read small writing on a tshirt. Thus, to complete the seeker metric, we decided that distance scores would only count towards the minimum distance matrix if the actor had moved her/himself some distance within the 5-s interval. Since this was still somewhat of an exploratory phase of metric development, we tried three variations of the seeker metric, based on the distance the actor was required to have moved in the last $5-\mathrm{s}$ in order to have minimum distances $d_{\min }$ count toward the matrix: $0.1,0.5$, and $1-\mathrm{m}$. Finally, since we wanted higher scores to indicate more seeking behavior, we took the reciprocal of each score $(1 / x)$ in order to compute the final seeker score.
$s_{i}=\frac{N-1}{\sum_{j \neq i} \min _{t=2}^{180}\left(d_{i, j}^{t} \text { if } \Delta_{i}^{t, t-1} \geq d_{\text {min }}\right)}$

Marginality This metric was calculated by averaging the number of other nodes within the actor's personal radius across each time interval $t$ over the course of the social event; lower numbers of interactions reflect greater marginality. We specified two variations of the metric, one in which the personal radius $r_{p}$ was $2-\mathrm{m}$ and one in which it was $5-\mathrm{m}$. One problem with measuring marginality in this way is that participants in more populated trials will accumulate higher scores simply by virtue of their greater availability of other participants. Thus, to control for the size of the social event, the raw score was then divided by the total number of people present in the trial. Again, since we wanted higher scores to indicate more marginality, we took the reciprocal of each score $(1 / x)$ to compute the final marginality score:

$$
m_{i}=\frac{180}{\sum_{t=1}^{180} \frac{\sum_{i \neq j} 1 \text { if } d_{i, j}^{t} \leq r_{p}}{N-1}} .
$$

Diversity/homophily For this metric, we took a snapshot of each actor's coordinates at every $5-\mathrm{s}$ interval and recorded the number of other participants representing each racial group (Black, White, and Asian) within their personal radius. Similar to the marginality metric, we computed two variations of this metric, one with the personal radius set at $2-\mathrm{m}$ and one at $5 \mathrm{~m}$. Then, for each time interval, a diversity quotient (DQ) was calculated (Tawa, 2017). The DQ is calculated using an entropy index (Quillian \& Redd, 2009; Tawa, 2017), which provides a single value to reflect the imbalance or balance of racial group representation within each snapshot; a score of 0 indicates no diversity at all (e.g., a snapshot with six Asian peers out of six peers), and a maximal value is reached when the racial groups represented in the snapshot are as equally balanced as possible, given the pool of prospective peers. In the present study, only Black, Asian, and White peers were available for selection. Thus, a maximal entropy index within a snapshot for someone interacting with six peers would be achieved when they had two Black, two Asian, and two White in their radius. An entropy score was computed by taking the negative of the log of the proportion of each group, multiplying by the proportion itself, and summing the products (Shannon \& Weaver, 1949). An entropy index was calculated for each participant $i$ and was represented by the following equation, where $K$ is the number of racial groups available (in the case of this study, always three) and $p_{k i}$ is the proportion of peers of $i$ in the snapshot named from each group:

$d_{i}=-\sum_{k=1}^{K} p_{k i} \log p_{k i}$. 
One of the challenges with measuring entropy based on peer group diversity, particularly within relatively small pools of possible peers, is that one's entropy score is highly influenced by the total possible diversity within the available pool of peers. Very diverse contexts are much more likely to yield diverse interactions than are less diverse contexts, and thus high entropy values do not necessarily indicate a person's actual preference for interacting with diverse peers. Thus, following Tawa (2017), our solution was to compute a DQ by also calculating an entropy value for the entire group at each time interval and then dividing each participants' entropy score by the entropy score of each group, again at each time interval.

In addition to the four observed behavioral interactions and metrics, we computed two unobserved metrics that were residuals of portions of the calculations of the observed metrics: minimum distance (average of minimum distances to all other nodes) and movement proportion (proportion of time intervals per node with movement $>0.1-\mathrm{m}$ ). These variables were retained in case they might be of use in later analyses. To summarize, we produced a total of ten metrics: leader, three versions of seeker (at $0.1,0.5$, and $1-\mathrm{m}$ ), two versions of marginality (at 2 and 5-m), two versions of diversity/ homophily (at 2-m and 5-m), and two residual metrics (minimum distance and movement proportion).

\section{Data analysis}

Normalization of the interpersonal metrics We examined skewness and kurtosis and conducted a Shapiro-Wilk test of normality for each of the ten variations of metrics (eight variations of observed metrics, and two unobserved metrics). For Shapiro-Wilk tests of normality, significance levels of .05 or higher indicate significantly normal distributions. Only one metric, marginality at $5-m$, was significantly normal. All three seeker variations and both diversity/homophily variations were negatively skewed, whereas the leader, marginality (at $2-\mathrm{m}$ ), and both unobserved variable scores were positively skewed (see Table 1).

Next, we ran a series of transformations to normalize the skewed metrics. To compute transformations with negatively skewed data, all scores for the metric were first reverse-scored. Once the final metrics were determined, scores were then reverse-scored back, so that they were valenced in the correct direction. We progressed from logarithmic (base 10), square root, to cube root transformation (see Table 2). Once we had achieved normality for each metric, we did not progress to the next level.

Through this procedure, we were able to successfully transform the following metrics so that they distributed normally: leader $(\log )$, seeker at $1-\mathrm{m}(\log )$, marginality at $2-\mathrm{m}(\log )$, marginality at $5-\mathrm{m}$ (no transformation needed), diversity/ homophily at $2-\mathrm{m}$ (square root), diversity/homophily at 2 - $\mathrm{m}$ (cube root), and movement proportion (log). In general, the larger-distance versions of metrics appeared to distribute more normally; thus, for each metric with multiple distance versions, we retained the one with the highest distance. Seeker at 0.1 and $0.5-\mathrm{m}$ and the minimum-distance metric could not be normalized. The following five metrics were retained for the final analyses: leadership (log), seeker (at $1-\mathrm{m}$; log), marginality (at 5-m), diversity/homophily (at 5-m; cube root), and movement proportion $(\log )$.

Construct validity of interpersonal metrics Establishing the construct validity of behavioral measures presents a paradox, given that self-report measures do not reliably relate to actual behavior (Baumeister et al., 2007; Blascovich et al., 2002; Sechrest, 2005); in fact, this line of reasoning is precisely why the development of direct measures of actual behavior is necessary (Baumeister et al., 2007). Yet, once a behavioral measure is proposed, it is necessary to understand how the new measure relates (or does not relate) to previously existing and established measures, in order to more fully understand this variable within the community of scholarship in which it now exists and will be used (Sechrest, 2005). In our present dataset, participants completed selfreport measures of intergroup attitudes after participating in the Second Life events. In this section, we examine the relation of our metrics to these measures: outgroup comfort (Cole \& Yip, 2008) and intergroup anxiety (Stephan \& Stephan, 1985). We also examine the intercorrelations between the proposed metrics. Although we tolerated some overlap between metrics, our intent here was to examine redundancy that would be characterized by any one measure correlating very strongly with all other measures (Hemphill, 2003; Tabachnick \& Fidell, 1996).

Outgroup comfort was measured by Cole and Yip's (2008) Outgroup Comfort Scale (OCS), which was originally used to assess Black students' state-based comfort, specifically in interaction in predominantly White social contexts (e.g., primarily White college settings). In this administration, we used race-neutral language to assess the outgroup comfort levels of multiple racial groups. A sample item from the OCS is "Settings with predominantly people of other races do not threaten me." With the present sample, the internal reliability for this scale was strong $(a=.92)$. Consistent with the conceptualization of our constructs described in the qualitative metric development section, we expected that seeker, marginality, and movement proportion would be negatively correlated, and that leadership and diversity/homophily would be positively correlated with outgroup comfort.

Intergroup anxiety was measured by the Intergroup Anxiety scale (IGA; Stephan \& Stephan, 1985). The IGA 
Table 1 Initial descriptives and normality tests for each metric variation

\begin{tabular}{|c|c|c|c|c|c|c|}
\hline & $M$ & $S D$ & $\mathrm{Sk}(S E)$ & $\mathrm{Kt}(S E)$ & $\begin{array}{l}\mathrm{S}- \\
\mathrm{W}\end{array}$ & Sig. \\
\hline Leader & 82.24 & 66.16 & $1.66(0.25)$ & $3.10(0.50)$ & .850 & .000 \\
\hline Seeker $(0.1-m)$ & 9.82 & 1.54 & $-3.33(0.25)$ & $18.27(0.50)$ & .741 & .000 \\
\hline Seeker $(0.5-m)$ & 10.49 & 1.85 & $-2.83(0.25)$ & $12.25(0.50)$ & .761 & .000 \\
\hline Seeker $(1-\mathrm{m})$ & 10.33 & 1.86 & $-2.27(0.25)$ & $9.77(0.50)$ & .836 & .000 \\
\hline Marginality $(2-m)$ & 0.071 & 0.037 & $1.44(0.25)$ & $3.39(0.50)$ & .890 & .000 \\
\hline Marginality $(5-m)$ & 0.315 & 0.090 & $-0.39(0.25)$ & $0.15(0.50)$ & .987 & .528 \\
\hline Diversity $(2-m)$ & 0.715 & 0.288 & $-1.03(0.25)$ & $0.39(0.50)$ & .893 & .000 \\
\hline Diversity $(5-m)$ & 0.876 & 0.195 & $-1.30(0.25)$ & $1.25(0.50)$ & .843 & .000 \\
\hline Movement & 69.09 & 58.57 & $1.83(0.25)$ & $3.88(0.50)$ & .829 & .000 \\
\hline Minimum distance & 1.96 & 1.05 & $4.57(0.25)$ & $31.17(0.50)$ & .640 & .000 \\
\hline Movement prop. & .113 & .065 & $1.05(0.25)$ & $1.65(0.50)$ & .937 & .000 \\
\hline
\end{tabular}

Bold shows the only nonsignificant result. $M=$ means; $S D=$ standard deviation; $\mathrm{Sk}(S E)=$ skewness and standard error of the skewness statistics; $\mathrm{Kt}(S E)$ = kurtosis and standard error of the kurtosis statistic; $\mathrm{S}-\mathrm{W}=$ Shapiro-Wilk test of normality statistics; Sig. = significance level of the S-W test statistic

is widely used to assess affective arousal that people tend to feel in cross-racial interactions in general, and not specific to settings in which one is a minority. On the IGA, participants are asked to rate on a scale of 1 to 10 whether they would feel more or less comfortable, awkward, self-conscious, happy, accepted, confident, irritated, impatient, defensive, suspicious, and careful when interacting with members of another racial group, as compared to their own. With the present sample, the internal reliability estimate was again strong $(a=.88)$. We expected that seeker behavior, marginality, and movement proportion would be positively correlated, and that leadership and diversity/ homphily would be negatively correlated with intergroup anxiety.

\section{Quantitative results}

Intercorrelations between behavioral metrics Bivariate correlations were run among all new metrics and the two self-report indices (see Table 3). With the exception of movement proportion, all of our predictions related to intergroup anxiety were confirmed, and none of our predictions related to outgroup comfort were confirmed. In most cases, intercorrelations between the behavioral metrics suggested some expected overlap. Predictably, those who did more seeking also tended to interact more with diversity and also tended to display less marginalized behavior. Less intuitively, those who did more seeking also tended to exhibit leadership behavior. The movement proportion measure strongly positively

Table 2 Normalization of metric variations

\begin{tabular}{|c|c|c|c|c|c|c|c|c|}
\hline & \multicolumn{2}{|c|}{ No Trans. } & \multicolumn{2}{|c|}{ Log (base 10) } & \multicolumn{2}{|c|}{ Square Root } & \multicolumn{2}{|c|}{ Cube Root } \\
\hline & S-W & Sig. & S-W & Sig. & S-W & Sig. & S-W & Sig. \\
\hline Leader & .850 & .000 & .980 & .190 & & & & \\
\hline Seeker $(0.1-\mathrm{m})$ & .741 & .000 & .968 & .029 & .894 & .000 & .853 & .000 \\
\hline Seeker $(0.5-\mathrm{m})$ & .761 & .000 & .970 & .040 & .902 & .000 & .871 & .000 \\
\hline Seeker $(1-\mathrm{m})$ & .836 & .000 & .989 & .269 & & & & \\
\hline Marginality $(2-m)$ & .890 & .000 & .972 & .050 & & & & \\
\hline Marginality $(5-m)$ & .987 & .528 & & & & & & \\
\hline Diversity $(2-m)$ & .893 & .000 & .898 & .000 & .976 & .087 & & \\
\hline Diversity $(5-m)$ & .843 & .000 & .960 & .008 & .944 & .001 & .972 & .201 \\
\hline Movement & .829 & .000 & .988 & .616 & & & & \\
\hline Minimum dist. & .640 & .000 & .937 & .000 & .835 & .000 & .884 & .000 \\
\hline Movement prop. & .937 & .000 & .977 & .118 & & & & \\
\hline
\end{tabular}

Bold values show where normalization was achieved for a particular variable. S-W = Shapiro-Wilk test of normality statistics; Sig. = significance level of the S-W test statistic; No Trans. = no transformation (original value); log (base 10) = logarithmic transformation; Square root = square root transformation; Cube root $=$ cube root transformation 
Table 3 Pearson's correlation matrix for the retained metrics and selfreport variables

\begin{tabular}{|c|c|c|c|c|c|c|}
\hline & 12 & 3 & 4 & 5 & 6 & 7 \\
\hline 1. Leader & $-.43^{* *}$ & .10 & .05 & $.79^{* *}$ & -.02 & $-.22^{*}$ \\
\hline 2. Seeker & - & $-.21^{*}$ & $.33^{* *}$ & $.67^{* *}$ & -.13 & $-.32^{* * *}$ \\
\hline 3. Marginality & & - & .02 & -.12 & .01 & $.21^{*}$ \\
\hline 4. Diversity/homophily & & & - & $.23^{*}$ & -.12 & $-.22^{*}$ \\
\hline 5. Movement prop. & & & & - & .03 & -.18 \\
\hline 6. Outgroup comfort & & & & & - & $.31^{* *}$ \\
\hline 7. Intergroup anxiety & & & & & & - \\
\hline
\end{tabular}

${ }^{*} p<.05,{ }^{* *} p<.01$

correlated with all behavior metrics except marginality. In fact, the correlations between movement proportion and leader $(r=$ $.79, p<.01)$ and seeker $(r=.67, p<.01)$ are notably high (Tabachnick \& Fidell, 1996), suggesting that movement might not be an independent construct from seeking and leadership (Hemphill, 2003); thus, movement behavior was not examined further as an independent construct, but rather was used as a control variable in the further analyses.

Group differences in interpersonal metrics We examined how participants' gender, race, and immigration status were related to our metrics by using multivariate analyses of variance (MANOVAs) in which the four metrics (leader, seeker, marginality, and diversity/homophily) were included as dependent variables. The metrics did not differ significantly by participant gender for the overall model [Wilk's $\lambda=.93 ; F(4,83)=1.68$, $\left.p=.16, \eta_{\mathrm{p}}^{2}=.075\right]$; however, examination of the effects on individual metrics within the model suggests that gender did effect leadership scores $\left[F(1,83)=4.01, p<.05, \eta_{\mathrm{p}}^{2}=.045\right]$. Males $(M=1.86, S D=0.41)$ had higher leadership scores than did females $(M=1.72, S D=0.38)$. Metrics did not differ significantly by participant race [Wilk's $\lambda=.95 ; F(8,164)=0.53$, $\left.p=.833, \eta_{\mathrm{p}}^{2}=.025\right]$ or immigration status [Wilk's $\lambda=.96$; $\left.F(4,83)=0.883, p=.478, \eta_{\mathrm{p}}^{2}=.041\right]$. Pearson's correlations determined that age was unrelated to any of the four metrics.

\section{Application of our metrics to a research question}

In this final section, we apply our metrics to an analysis considering the effects of resource competition on racial group members' interpersonal behavior. Our purpose for this application study was to demonstrate how our metrics can provide us with understandings of interpersonal interactions above and beyond the relatively rudimentary spatiotemporal metrics that have characterized previous studies (e.g., Tawa et al., 2015; Toppenberg et al., 2015). In fact, in a previous analysis of these same data (Tawa et al., 2015), we were limited in our understanding of how resource competition affected intergroup relations because of our sole reliance on proxemics. In this previous analysis, we found that within resource competition settings, Black and Asian participants initially approached each other, but then began to move away over the duration of the event (Tawa et al., 2015). Although these findings supported our prediction that resource competition would cause greater distance between Black and Asian participants, we also raised questions about the more precise mechanisms through which resource competition gives rise to increasing physical distance (Tawa et al., 2015). For example, it is possible that conditions of resource competition give rise to people feeling marginalized from others, which in turn could lead to more ingroup isolating behavior. Given that our metrics emerged from the qualitative analysis in this article, and were thus not available to us a priori, our examination of the potential mediating roles of our metrics is exploratory and not hypothesized.

In the present analyses, physical distance scores were calculated as the total of a participant's (e.g., a Black participant) scores to both outgroups (e.g., Whites and Asians); including physical distance scores in our present analyses enabled us to evaluate how our new metrics performed in comparison. Change in distances over time was determined by computing the average rate of change between outgroup physical distance scores across each of the three 5-min time blocks comprising the 15 -min social event. Thus, the outcome variable is a single score that reflects a change in physical distance toward outgroup members over the duration of the trial.

Participants The participants included 131 (male $=40$, female $=91$ ) people recruited online and at the first author's previous university; the participants self-identified as Black $(n=26)$, Asian $(n=34)$, or White $(n=71)$. Twenty-five of our participants were born outside the United States, and the average age was 24.11 years $(S D=7.82)$. The online recruiting included postings on social networking sites (e.g., Facebook) and classified advertisement sites (e.g., Craigslist). The university recruitment included flyers, blast emails, class announcements, and the undergraduate subject pool. Participants were then randomly assigned to a resource competition condition ( $n$ $=55)$ or a utopian condition $(n=68)$. The multiple recruitment strategies, broad geographic pool, and random group assignment minimized the likelihood of participants knowing each other prior to participation. The participants were relatively successful in creating avatars that resembled themselves racially, assessed both via self-report and through research assistants' objective ratings of headshots of the avatars (see Tawa, 2017, for more detail).

Preliminary analyses An analysis of variance confirmed that there was a higher average rate of change in outgroup distance in the resource competition condition $(M=2.32, S D=1.96)$ than in the utopian condition $(M=-0.56, S D=2.96)$ and that this difference was significant $[F(1,87)=29.86, p<.01, d=$ 1.15]. In actual terms, within the resource competition 
condition, participants moved an average of 2.32-m away from outgroup members during the social events, whereas during the utopian condition, participants moved an average of $0.56-\mathrm{m}$ toward outgroup members.

With regard to the metrics developed in this study, participants did more seeking in the resource competition $(M=.71$, $S D=.19)$ than in the utopian condition $(M=.61, S D=.21)$, and this difference was significant $[F(1,87)=5.11, p<.05, d$ $=0.50]$. Participants also interacted with more diversity in the resource competition condition $(M=.51, S D=.10)$ than in the utopian condition $(M=.32, S D=.19)$, and this difference was also significant $[F(1,87)=33.47, p<.01, d=1.25]$. Resource competition was unrelated to leader, marginality, or movement behavior.

Bivariate correlations determined that the average rate of change in outgroup distance was positively correlated with marginality $(r=.41, p<.01)$ and diversity/homophily $(r=$ $.45, p<.01)$. In the final analysis, we ran four separate mediation models examining the potential mediating effect of each metric-leadership, seeker, marginality, and diversity/ homophily - on the relationship between resource competition and the average rate of change in physical distance.

Mediation analyses We examined the potential for each observed metric to mediate the relationship between the presence of resource competition and greater movement away from outgroup members. Mediation analyses were examined using a bootstrapping method aided by PROCESS version 2 , model number 4 (Hayes, 2013). Bootstrapping provides an estimate of both the direct path (i.e., the relation between the predictor and outcome variable while controlling for the effect of the mediation variable) and the indirect path (i.e., the path from the predictor to the outcome through the mediation variable). Each analysis was based on 10,000 resamples of the dataset with a bias-corrected $95 \%$ confidence interval. In this method, the indirect effect is considered significant at $p<.05$ if the provided confidence interval does not contain the value of 0 (Hayes, 2013; Preacher \& Hayes, 2008). The PROCESS macro allows multiple mediators to be tested simultaneously in order to tease apart the potentially overlapping effect of confounded variables. Thus, we were able to control for the potentially competing effects of correlated metrics within each model.

Seeker behavior was examined as a mediator on the relationship between resource competition and increases in outgroup distance, while controlling for movement, marginality, leadership, and interaction with diversity (given that seeker was correlated with each of these variables). This indirect path was not significant (indirect effect $=-0.28, \mathrm{CI}[-0.91$, $0.22])$. Leader behavior was examined as a mediator while controlling for movement and seeker behavior. This indirect path was not significant (indirect effect $=0.27$, CI $[-0.30$, 1.27]). Marginality was examined as a mediator while controlling for seeker behavior. This indirect path was not significant (indirect effect $=-0.02$, CI $[-0.59,0.67]$ ). Finally, diversity/homophily was examined as a mediator while controlling for movement and seeker behavior. This indirect path was significant (indirect effect $=0.76, \mathrm{CI}[0.15$, $1.88]$ ), suggesting that interaction with diversity may operate as a mechanism through which resource competition leads to increases in outgroup physical distance.

\section{Discussion}

Although our primary intent for the application study was to demonstrate how our metrics could be applied to research problems, the findings from this study warrant some brief discussion. Following this discussion of research findings, we turn to a more general discussion in which we address some methodological strengths and limitations of our work, focusing on the qualitative component and the validity of our study and metrics. Next, we consider some possible applications of our metrics for future psychological research studies and address how these future applications can contribute to APA's call for increasing the production of research that directly assesses behavior, rather than primary relying on selfreport methods (Baumeister et al., 2007).

\section{Discussion of findings from the application study}

Our finding that resource competition is related to increases in physical distance from outgroup members is consistent with findings specific to Blacks and Asians in Tawa et al. (2015). Yet our metrics provide us further insight into how resource competition affects physical distance. In our preliminary analyses, we found both higher seeker scores and higher interaction with diversity scores in resource competition than in utopian conditions. It appears that those of our participants who believed they were competing for resources took more of a "survey" approach to getting to know others in the social event rather than indulging fewer peer interactions at greater depth. Participants in resource competition conditions may have tended to favor briefer interactions with greater numbers of people, perhaps in an effort to evaluate as many people as possible in order to maximize their chances of establishing a peer group with the highest levels of "intelligence" and "hard work" ethic. Perhaps though, given the limited time in which participants had to make their friendship selections, participants in the resource competition condition were more likely to rely on superficial markers from which to draw assumptions about people's character, for example, skin color or perceived racial membership. Previous research does suggest that more visible individual differences (such as gender and race) tend to play a particularly important role in the peer selection process (de Klepper et al., 2010; van de Bunt et al., 1999; van Duijn et al., 2003). 
Moreover, our mediation analyses suggest that this interaction with diversity is the operative mechanism through which resource competition affects increases in outgroup distance. We found that people interacted with more diversity in resource competition conditions than in utopian conditions, and in turn, interaction with diversity was related to greater increases in distance from outgroup members over the course of the trial. It appears that the nature of the interactions with diversity in resource competition may have been deterring in some way. One possibility is that people might have tended to interact with outgroup members as a way of scrutinizing their level of competence in intelligence and/or work ethic, or perhaps they were even attempting to discern others' racial group membership if it was not immediately apparent. Perhaps greater distancing occurred as a result of these more discerning or superficial interactions with outgroup members. These findings support classic social psychology theory, which predicts that group interactions will result in adverse outcomes when groups believe themselves to be in competition with other groups for limited resources (Blumer, 1958).

\section{General discussion}

In this article, we used a sequential qualitative $\rightarrow$ quantitative mixed-method procedure to derive and normalize four metrics of interpersonal behavior from continuous spatiotemporal data. We then applied our metrics to a research problem related to the influence of resource competition on interpersonal behavior among racial groups. Our metrics were developed specifically for continuous-time, microspatial, and egocentricperspective data, which we believe are likely to be of most interest to social and intergroup psychologists.

Each of our interpersonal behaviors-leadership, seeker, marginality, and diversity/homophily - initially emerged from qualitative observations of patterns of movements among avatars interacting in social events in the virtual environment Second Life. In the qualitative analyses, the movement of avatars throughout the 15-min trials was triangulated with discussions recorded in chat logs. These conversational processes provided an important view into the actual movements during the trials across time; we were able to observe how participants' conversational agency affected their movements within the virtual space, as well as that of others. In turn, we saw in some cases, the ways that the movements of key actors, as evinced first by their conversational strategies, influenced the movements of others. Insights gained from the chat logs, along with visual analysis of dynamic spatiotemporal interactions, were crucial for making sense out of the changes that we observed and measured over time. Indeed, in recent years, network analysts have called for greater and improved integration of qualitative and quantitative analytics in social network research (Dominguez \& Hollstein, 2014).
One reasonable challenge to our study is related to the validity of virtual worlds: Are people's behaviors in virtual environments analogous to people's behaviors in real life? Whether or not virtual-world behavior seems to "map" onto real-life behavior seems to depend on the type of behavior under study; for example, behaviors motivated by fear of physical pain or death do not map well (Williams, 2010). On the other hand, virtual behaviors-such as physical movement behavior - that is motivated by emotional discomfort may map more strongly onto real-life behavior (Williams, 2010). Indeed, a relative wealth of studies support a consistency between virtual interpersonal behavior - such as interpersonal distancing and approach-and real-life behavior (Bailenson et al., 2004; Bailenson et al., 2008; Blascovich et al., 2002; Dotsch \& Wigboldus, 2008; Eastwick \& Gardner, 2009; Feldon \& Kafai, 2008; Friedman et al., 2007; Hasler \& Friedman, 2012; Lee \& Park, 2011; Lo, 2008; McCall et al., 2009; Tawa, 2017; Tawa et al., 2015; Toppenberg et al., 2015; Vang \& Fox, 2014; Yee \& Bailenson, 2008; Yee et al., 2007).

Another common challenge to the validity of virtual behaviors is referred to as the "online disinhibition effect" (Suler, 2004), which suggests that people are likely to be less concerned about the impact of their behaviors on others because of the anonymity provided by online environments. For example, people may tend to be more explicitly racist or prejudiced online. We did not see any evidence of explicit racism or prejudice in our qualitative analyses; in fact, consistent with real life, the avatars tended to use codified strategies for trying to determine others' race, perhaps because asking directly about race may be seen as inappropriate (Apfelbaum, Sommer, \& Norton, 2008). Nonetheless, this is a reasonable challenge, and future research should continue to contribute to the developing body of research examining the relationship between real-life and virtual behaviors (Bayraktar \& Amca, 2012; Bailenson et al., 2008; Blascovich et al., 2002; Dotsch \& Wigboldus, 2008; Eastwick \& Gardner, 2009; Feldon \& Kafai, 2008; Lee \& Park, 2011; Lo, 2008; Tawa, 2017; Tawa et al., 2015; Vang \& Fox, 2014; Yee \& Bailenson, 2008; Yee et al., 2007).

In general, we recognize that within experimental research, there is an inherent tension between ecological validity and developing tightly controlled environments (Blascovich et al., 2002); as one introduces the greater nuances and variability that are present in real life (ecological validity), one simultaneously compromises laboratory control and statistical power. Virtual environments may actually provide a unique solution to this problem; on the one hand, they offer highly detailed social environments and personifications, while simultaneously they can be held constant over multiple experimental trials and studies. As such, the virtual environment may actually be an ideal medium through which metrics of interpersonal patterns of behavior are initially explored and derived. 
Our metric development is based on group interactions that are relatively short in duration (i.e., 15-min). We see both pros and cons to developing metrics from these short interactions. Certainly, a 15-min encounter is likely to be shorter than a typical interactive group event, such as an educational class period, a social gathering, or board meeting, thus compromising real-life validity. On the other hand, the relatively contained duration allowed us to more easily make observations about the emerging roles of the individuals and subgroups across multiple trials. Furthermore, a recent metaanalysis has demonstrated that group-specific behavioral norms and roles within a group often emerge very early on in the group process - as early as the first 15-20-min - and that these norms and roles are remarkably predictive of the final established norms and roles for the remainder of the group process (Allen \& O’Neill, 2015).

We made some effort to establish the construct validity of our interpersonal behavior metrics. Actual behaviors, however, frequently do not correlate with self-report behavior, at least in part because self-report is often plagued by socialdesirability effects (Crowne \& Marlowe, 1960; Freeman, Romney, \& Freeman, 1987). Nonetheless, we did find that our metrics were related to one self-report attitudeintergroup anxiety - in the directions that were predicted. People who self-reported being anxious in intergroup settings tended to have lower leadership, seeker, and diversity scores, and higher marginality scores. All of our metrics were unrelated to outgroup discomfort. It is possible that because the outgroup comfort measure was originally developed to assess the experience of comfort in settings in which one is in a numerical minority, this construct had less relevance to our relatively numerically diverse settings, particularly among White participants, who were never a minority within any trial. Intergroup anxiety is a more stable, trait-based construct, and presumably it measures anxiety experienced during intergroup interactions more generally (Cole \& Yip, 2008; Stephan $\&$ Stephan, 1985). In addition, we found that males had marginally higher leadership scores. Our analysis of videos and chats were gender-blind, in that we did not visually mark gender in the videos, and the IDs in the chats did not by default contain gender information. In this way, the leadership metric would not have been influenced by any stereotyped notions about gender differences in leadership behavior. That said, the leadership metric may be most sensitive to stereotypically masculine leadership behavior, namely behavior that is more authoritative and less collaborative (Eagly \& Johnson, 1990; e.g., "points" went to actors who remained relatively still while others approached them).

Intercorrelations between metrics also contribute to our understanding of these emerging metrics. It is intuitive, for example, that seeking and diversity behaviors are somewhat related, as both comprise interacting with a wider variety of people. It is less intuitive that leader and seeker behavior would be related, given that we conceptualize seekers as frequently moving around, whereas our operationalization of leadership rewarded nodes with points for being stationary and having other nodes approach them. Yet, perhaps in the context of short-term tasks seekers become more quickly known to the group, and thus develop an attractive quality that brings people toward them. Future research should continue to explore the relationship between leading and seeking behavior

In the application of our metrics to a research problem, we found that when people believe they are competing for resources, they tend to move away from racial outgroup members over time. Comparably, in social settings absent of resource competition (such as the group interactions depicted in Fig. 1), people tend to move toward outgroup members over time. We established a similar finding in a previous analysis of the data, specific to Blacks' and Asians' physical distance (Tawa et al., 2015). Yet, given that physical proximity is a relatively rudimentary measure of interpersonal behavior, and could, for example, indicate either warmth or suspicion (e.g., Ashton et al., 1980), we were limited in our interpretation of the findings. We have presented this case study in order to demonstrate the potential usefulness of our metrics. For instance, in our application study we also found that resource competition contexts were also related to greater seeking behavior and greater interaction with diversity than in utopian conditions. When monetary reward is at stake, people seem to be motivated to talk to more people at less depth (rather than fewer people at more depth), in order to maximize the chance of finding ideal group members. Our metrics allowed us to understand that such "shallow" interactions-particularly when with diverse outgroup members - are one mechanism that ultimately leads to greater distance from outgroup members, perhaps because they are seen as superficial and are thus off-putting.

Technology will increasingly enable the collection of continuous-time, microspatial, and egocentric-perspective data with "real-life" human participants simultaneously interacting in a shared space. To be clear, the technology for collecting these data already exists - for example, the high-resolution GPS personal activity trackers (Ákos et al., 2014) and transponders with base stations (Frencken et al., 2011) described earlier in our review. Other promising technologies exist as well, including radio frequency identification (RFID), which has a sensing distance of 1-2-m (Cattuto et al., 2010), and MIT's "sociometric badges," which use Bluetooth sensors and can record the distances between any two actors wearing badges within less than $1 \mathrm{~m}$ and up to $10 \mathrm{~m}$ (Choudhury \& Pentland, 2003). Yet these devices are frequently quite expensive, and granting agencies are unlikely to fund research proposing to use them without a strong understanding of how to analyze these data. Thus, our primary goal for this research was to give interested intergroup and social psychology 
researchers a starting point for how to begin to reduce the vast amounts of raw data derived from these devices into useable individual-level metrics that can be, for example, correlated with standard survey measures or other individual-level variables that are more familiar to psychological researchers.

These technologies and the methods for analyzing their data will open up a realm of possibility for psychological inquiry. For example, in our research we found that within competitive resource contexts, brief interactions with diverse others can be detrimental to interpersonal interactions, insofar as they may appear to be superficial; yet we wonder about how interactions with diverse others over a much more sustained encounter, such as interactions with coworkers, can be related to positive interpersonal interactions and intergroup relations. Within the workplace setting, researchers may also consider how the various roles depicted in the metrics are related to productivity and the overall success of a working group; perhaps there is an ideal combination of leaders, seekers, and even those on the margins that comprises the most productive working groups. Additionally, it would be valuable to understand both situational and psychological predictors of these interpersonal behaviors. As examples, one could experimentally examine how various teaching methods (e.g., didactic vs. interactive) relate to students' behavioral marginalization in the classroom, examine the processes that lead to the emergence of student leaders during group project sessions, and examine the personality correlates or predictors of seekers.

Finally, future researchers might be interested in adopting our approach of using virtual environments to observe and generate metrics of other types of nonverbal, interpersonal behavior for real-world applications. Virtual environments have already been successfully used to track behaviors such as eye contact (Bailenson, Blascovich, Beall, \& Loomis, 2001), body orientation (Friedman et al., 2007), and physical aggression (McCall et al., 2009). The relative ease and costefficiency of virtual technology, coupled with the acuity of the behavioral user data that it offers, provides an ideal medium for the exploration and development of measurement of behavior over time (Williams, 2010). Continuing to develop our ability to measure behavior over time will contribute to a long overdue response to APA's call for more research examining real-time behaviors (Baumeister et al., 2007).

\section{References}

Aharony, N., Pan, W., Ip, C., Khayal, I., \& Pentland, A. (2011). Social fMRI: Investigating and shaping social mechanisms in the real world. Pervasive and Mobile Computing, 7, 643-659.

Ákos, Z., Beck, R., Nagy, M., Vicsek, T., \& Kubinyi, E. (2014). Leadership and path characteristics during walks are linked to dominance order and individual traits in dogs. PLoS Computational
Biology, 10, e1003446. https://doi.org/10.1371/journal.pcbi. 1003446

Allen, N. J., \& O'Neill, T. A. (2015). The trajectory of emergence of shared group-level constructs. Small Group Research, 46, 352-390.

Allport, G. (1985). The historical background of social psychology. In G. Lindzey \& E. Aronson (Eds.), Handbook of social psychology (3rd ed., pp. 1-46). New York, NY: Random House.

Andersson, M., Gudmundsson, J., Laube, P., \& Wolle, T. (2008). Reporting leaders and followers among trajectories of moving point objects. Geoinformatica, 12, 497-528.

Andrienko, N., \& Andrienko, G. (2007). Designing visual analytics methods for massive collections of movement data. Cartographica, 42, 117-138. https://doi.org/10.3138/carto.42.2.117

Apfelbaum, E. P., Sommer, S. R., \& Norton, M. I. (2008). Seeing race and seeming racist? Evaluating strategic colorblindness in social interaction. Journal of Personality and Social Psychology, 95, 918-932.

Ashton, N. L., Shaw, M. E., \& Worsham, A. P. (1980). Affective reactions to interpersonal distances by friends and strangers. Bulletin of the Psychonomic Society, 15, 306-308.

Bailenson, J. N., Aharoni, E., Beall, A. C., Guadagno, R. E., Dimov, A., \& Blascovich, J. (2004, October). Comparing behavioral and selfreport measures of embodied agents' social presence in immersive virtual environments. Paper presented at the 7 th Annual International Workshop on PRESENCE, Valencia, Spain.

Bailenson, J. N., Blascovich, J., Beall, A. C., \& Loomis, J. M. (2001). Equilibrium theory revisited: Mutual gaze and personal space in virtual environments. Presence: Teleoperators and Virtual Environments, 10, 583-598.

Bailenson, J. N., Blascovich, J., \& Guadagno, R. E. (2008). Selfrepresentations in immersive virtual environments. Journal of Applied Social Psychology, 38, 2673-2690.

Baumeister, R. F., Vohs, K. D., \& Funder, D. C. (2007). Psychology as the science of self-reports and finger movements: Whatever happened to actual behavior? Perspectives on Psychological Science, 2, 396403. https://doi.org/10.1111/j.1745-6916.2007.00051.x

Bayraktar, F., \& Amca, H. (2012). Interrelations between virtual-world and real-world activities: Comparison of genders, age groups, and pathological and nonpathological Internet users. Cyberpsychology, Behavior, and Social Networking, 15, 263-269. https://doi.org/10. 1089/cyber.2011.0337

Benkert, M., Gudmundsson, J., Hübner, F., \& Wolle, T. (2008). Reporting flock patterns. Computational Geometry, 41, 111-125.

Bian, L. (2000). Component modeling for the spatial representation of wildlife movements. Journal of Environmental Management, 59, 235-245.

Blascovich, J., Loomis, J., Beall, A. C., Swinth, K. R., Hoyt, C. L., \& Bailenson, J. N. (2002). Immersive virtual environment technology as a methodological tool for social psychology. Psychological Inquiry, 13, 103-124.

Blumer, H. (1958). Race prejudice as a sense of group position. Pacific Sociological Review, 1, 3-7.

Cattuto, C., Van den Broeck, W., Barrat, A., Colizza, V., Pinton, J.-F., \& Vespignani, A. (2010). Dynamics of person-to-person interactions from distributed RFID sensor networks. PLoS ONE, 5, e11596. https://doi.org/10.1371/journal.pone.0011596

Cheryan, S., \& Bodenhausen, G. V. (2000). When positive stereotypes threaten intellectual performance: The psychological hazards of "model minority" status. Psychological Science, 11, 399-402.

Cho, E., Mayers, S. A., \& Leskovec, J. (2011). Friendship and mobility: User movement in location-based social networks. In Proceedings of the 17th ACM SIGKDD International Conference on Knowledge Discovery and Data Mining (pp. 1082-1090). New York, NY: ACM Press.

Choudhury, T., \& Pentland, A. (2003). Sensing and modeling human networks using the sociometer. In Proceedings of the 7th IEEE 
International Symposium on Wearable Computers, ISWC '03 (p. 216). Washington, DC: IEEE Computer Society.

Cole, E. R., \& Yip, T. (2008). Using outgroup comfort to predict Black college students' experiences. Cultural Diversity and Ethnic Minority Psychology, 14, 57-66.

Creswell, J. W., \& Clark, V. L. P. (2017). Designing and conducting mixed methods research. Thousand Oaks, CA: Sage.

Crowne, D. P., \& Marlowe, D. (1960). A new scale of social desirability independent of psychopathology. Journal of Consulting Psychology, 24, 349-354.

de Klepper, M., Sleebos, E., van de Bunt, G., \& Agneessens, F. (2010). Similarity in friendship networks: Selection or influence? The effects of constraining contexts and non-visible individual attributes. Social Networks, 32, 82-90.

Dijkstra, J. K., Cillessen, A. H. N., \& Borch, C. (2013). Popularity and adolescent friendship networks: Selection and influence dynamics. Developmental Psychology, 49, 1242-1252.

Dodge, S., Weibel, R., Ahearn, S. C., Buchin, M., \& Miller, J. A. (2016). Analysis of movement data. International Journal of Geographical Information Science, 30, 825-834

Dodge, S., Weibel, R., \& Lautenschütz, A.-K. (2008). Towards a taxonomy of movement patterns. Information Visualization, 7, 240-252.

Dominguez, S., \& Hollstein, B. (Eds.). (2014). Mixed methods social networks research: Design and applications. Cambridge, UK: Cambridge University Press.

Dotsch, R., \& Wigboldus, D. H. J. (2008). Virtual prejudice. Journal of Experimental Social Psychology, 44, 1194-1198.

Duarte, R., Araújo, D., Freire, L., Folgado, H., Fernandes, O., \& Davids, K. (2012). Intra- and inter-group coordination patterns reveal collective behaviors of football players near the scoring zone. Human Movement Science, 31, 1639-1651.

Ducheneaut, N., Yee, N., Nickell, E., \& Moore, R. J. (2007). The life and death of online gaming communities: A look at guilds in World of Warcraft. In Proceedings of the 2017 CHI Conference on Human Factors in Computing Systems (pp. 839-848). New York, NY: ACM Press.

Eagle, N., Pentland, A., \& Lazer, D. (2009). Inferring friendship network structure by using mobile phone data. Proceedings of the National Academy of Sciences, 106, 15274-15278.

Eagly, A. H., \& Johnson, B. T. (1990). Gender and leadership style: A meta-analysis. Psychological Bulletin, 108, 233-256. https://doi. org/10.1037/0033-2909.108.2.233

Eastwick, P. W., \& Gardner, W. L. (2009). Is it a game? Evidence for social influence in the virtual world. Social Influence, 4, 18-32.

Evans, G. W., \& Howard, R. B. (1973). Personal space. Psychological Bulletin, 80, 334-344.

Feldon, D. F., \& Kafai, Y. B. (2008). Mixed methods for mixed reality: Understanding users' avatar activities in virtual worlds. Educational Technology Research and Development, 56, 575-593.

Freeman, L., Romney, A., \& Freeman, S. (1987). Cognitive structure and informant accuracy. American Anthropologist, 89, 310-325.

Frencken, W. G. P., Lemmink, K. A. P. M., Delleman, N. J., \& Visscher, C. (2011). Oscillations of centroid position and surface area of soccer teams in small-sided games. European Journal of Sport Science, $11,215-223$

Friedman, D., Steed, A., \& Slater, M. (2007). Spatial social behavior in Second Life. In Proceedings of the International Workshop on Intelligent Virtual Agents (pp. 252-263). Berlin, Germany: Springer.

Glaser, B. G., Strauss, A. L., \& Strutzel, E. (1968). The discovery of grounded theory: Strategies for qualitative research. Nursing Research, 17, 364

Hall, E. T. (1964). Silent assumptions in social communication. Disorders of Communication, 42, 41-55.

Hasler, B. S., \& Friedman, D. A. (2012). Sociocultural conventions in avatar-mediated nonverbal communication: A cross-cultural analysis of virtual proxemics. Journal of Intercultural Communication Research, 41, 238-259.

Hayes, A. F. (2013). Methodology in the social sciences. Introduction to mediation, moderation, and conditional process analysis: A regression-based approach. New York, NY: Guilford Press.

Hemphill, J. F. (2003). Interpreting the magnitude of correlation coefficients. American Psychologist, 58, 78-80.

Hurvitz, P. M., Moudon, A. V., Kang, B., Saelens, B. E., \& Duncan, G. E. (2014). Emerging technologies for assessing physical activity behaviors in space and time. Frontiers in Public Health, 2, 2:1-15. https://doi.org/10.3389/fpubh.2014.00002

Kossinets, G., \& Watts, D. J. (2009). Origins of homophily in an evolving social network. American Journal of Sociology, 115, 405-450.

Laube, P., Imfeld, S., \& Weibel, R. (2005). Discovering relative motion patterns in groups of moving point objects. International Journal of Geographical Information Science, 19, 639-668.

Lee, J., \& Park, S. (2011). "Whose second life is this?" How avatar-based racial cues shape ethno-racial minorities' perception of virtual worlds. Cyberpsychology, Behavior, and Social Networking, 14, 637-642.

Levitt, H. M., Bamberg, M., Creswell, J. W., Frost, D. M., Josselson, R., \& Suárez-Orozco, C. (2018). Journal article reporting standards for qualitative primary, qualitative meta-analytic, and mixed methods research in psychology: The APA Publications and Communications Board task force report. American Psychologist, 73, 26-46. https://doi.org/10.1037/amp0000151

Li, C. T., \& Lin, S. D. (2011, August). Social flocks: a crowd simulation framework for social network generation, community detection, and collective behavior modeling. In Proceedings of the 17th ACM SIGKDD International Conference on Knowledge Discovery and Data Mining (pp. 765-768). New York, NY: ACM Press.

Lo, S. (2008). The impact of online game character's outward attractiveness and social status on interpersonal attraction. Computers in Human Behavior, 24, 1947-1958.

Long, J. A., \& Nelson, T. A. (2013a). Measuring dynamic interaction in movement data. Transactions in Geographical Information Science, $17,62-77$.

Long, J. A., \& Nelson, T. A. (2013b). A review of quantitative methods for movement data. International Journal of Geographical Information Science, 27, 292-318.

McArdle, G., Demsar, U., van der Spek, S., \& McLoone, S. (2014). Classifying pedestrian movement behaviour from GPS trajectories using visualization and clustering. Annals of Geographic Information Science, 20, 85-98.

McCall, C., Blascovich, J., Young, A., \& Persky, S. (2009). Proxemic behaviors as predictors of aggression towards Black (but not White) males in an immersive social environment. Social Influence, 4, 138154.

Mehrabian, A. (1969). Significance of posture and position in the communication of attitude and status relationships. Psychological Bulletin, 71, 359-372.

Mercken, L., Steglich, C., Sinclair, P., Holliday, J., \& Moore, L. (2012). A longitudinal social network analysis of peer influence, peer selection, and smoking behavior among adolescents in British schools. Health Psychology, 31, 450-459.

Noyon, V., Claramunt, C., \& Devogele, T. (2007). A relative representation of trajectories in geographical spaces. Geoinformatica, 11, 479496.

Passos, P., Araújo, D., Davids, K., Gouveia, L., Serpa, S., Milho, J., \& Fonseca, S. (2009). Interpersonal pattern dynamics and adaptive behavior in multiagent neurobiological systems: Conceptual model and data. Journal of Motor Behavior, 41, 445-459.

Preacher, K. J., \& Hayes, A. F. (2008). Asymptotic and resampling strategies for assessing and comparing indirect effects in multiple mediator models. Behavior Research Methods, 40, 879-891. https://doi. org/10.3758/BRM.40.3.879 
Quillian, L., \& Redd, R. (2009). The friendship networks of multiracial adolescents. Social Science Research, 38, 279-295.

Rogers, E. M. (2003). Diffusion of innovations (5th ed.). New York, NY: Free Press.

Saelens, B. E., Moudon, A. V., Kang, B., Hurvitz, P. M., \& Zhou, C. (2014). Relation between higher physical activity and public transit use. American Journal of Public Health, 104, 854-859.

Sechrest, L. (2005). Validity of measures is no simple matter. Health Service Research, 40, 1584-1604.

Shannon, C. E., \& Weaver, W. (1949). Mathematical theory of communication. Urbana, IL: University of Illinois Press.

Sijtsema, J. J., Ojanen, T., Veenstra, R., Lindenberg, S., Hawley, P. H., \& Little, T. D. (2010). Forms and functions of aggression in adolescent friendship selection and influence: A longitudinal social network analysis. Social Development, 19, 515-534.

Silm, S., Ahas, R., \& Nuga, M. (2013). Gender differences in space-time mobility patterns in a postcommunist city: A case study based on mobile positioning in the suburbs of Tallinn. Environment and Planning B: Planning and Design, 40, 814-828.

Sorokowska, A., Sorokowski, P., Hilpert, P., Cantarero, K., Frackowiak, T., Ahmadi, K., . . . Pierce, J. D. J. (2017). Preferred interpersonal distances: A global comparison. Journal of Cross-Cultural Psychology, 48, 577-592. https://doi.org/10.1177/ 0022022117698039

Steglich, C., Snijders, T. A. B., \& West, P. (2006). Applying SIENA: An illustrative analysis of the coevolution of adolescents' friendship networks, taste in music, and alcohol consumption. Methodology, $2,48-56$.

Stephan, W. G., \& Stephan, C. W. (1985). Intergroup anxiety. Journal of Social Issues, 41, 157-175.

Suler, J. (2004). The online disinhibition effect. CyberPsychology and Behavior, 7, 321-326.

Tabachnick, B. G., \& Fidell, L. S. (1996). Using multivariate statistics (3rd ed.). New York, NY: Harper Collins.

Tantipathananandh, C., Berger-Wolf, T., \& Kempe, D. (2007). A framework for community identification in dynamic social networks. In Proceedings of the 13th ACM SIGKDD International Conference on Knowledge Discovery and Data Mining (pp. 717-726). New York, NY: ACM Press.

Tawa, J. (2017). Asymmetric peer selections among Blacks, Asians, and Whites in a virtual environment: Preliminary evidence for triangulated threat theory. Journal of Social Psychology, 157, 736753. https://doi.org/10.1080/00224545.2017.1294140

Tawa, J., Negrón, R., Suyemoto, K. L., \& Carter, A. S. (2015). The effects of resource competition on Blacks', Asians', and Whites' social distances: A virtual world method. Group Processes and Intergroup Relations, 18, 761-777.

Toppenberg, H. L., Bos, A., Ruiter, R., Wigboldus, D., \& Pryor, J. (2015). HIV-related stigma in social interactions: Approach and avoidance behaviour in a virtual environment. European Journal of Social Psychology, 45, 169-179.

van de Bunt, G. G., van Duijn, M. A. J., \& Snijders, T. A. B. (1999). Friendship networks through time: An actor-oriented dynamic statistical network model. Computational and Mathematical Organization Theory, 5, 167-192.

van Duijn, M. A. J., Zeggelink, E. P. H., Huisman, M., Stokman, F. N., \& Wasseur, F. W. (2003). Evolution of sociology freshmen into a friendship network. Journal of Mathematical Sociology, 27, 153191.

Vang, M. H., \& Fox, J. (2014). Race in virtual environments: Competitive versus cooperative games with Black or White avatars. Cyberpsychology, Behavior, and Social Networking, 17, 235-240.

Williams, D. (2010). The mapping principle, and a research framework for virtual worlds. Communication Theory, 20, 451-470.

Woolf, K., Potts, H. W. W., Patel, S., \& McManus, I. C. (2012). The hidden medical school: A longitudinal study of how social networks form, and how they relate to academic performance. Medical Teacher, 34, 577-586.

Yee, N., \& Bailenson, J. N. (2008). A method for longitudinal behavioral data collection in Second Life. Presence, 17, 594-596. https://doi. org/10.1162/pres.17.6.594

Yee, N., Bailenson, J. N., Urbanek, M., Chang, F., \& Merget, D. (2007). The unbearable likeness of being digital: The persistence of nonverbal social norms in online virtual environments. CyberPsychology \& Behavior, 10, 115-121.

Zhang, Y., \& Chai, Y. W. (2011). The spatio-temporal activity pattern of the middle and low-income residents in Beijing, China. Scientia Geographica Sinica, 9, 1056-1064.

Publisher's note Springer Nature remains neutral with regard to jurisdictional claims in published maps and institutional affiliations. 